Article

\title{
Sensory System for Implementing a Human-Computer Interface Based on Electrooculography
}

\section{Rafael Barea *, Luciano Boquete, Jose Manuel Rodriguez-Ascariz, Sergio Ortega and Elena López}

Department of Electronics, University of Alcalá, Alcalá de Henares 28871, Madrid, Spain; E-Mails: luciano.boquete@uah.es (L.B.); jmra@depeca.uah.es (J.M.R.-A.); sergioortegarecuero@hotmail.com (S.O.); elena@depeca.uah.es (E.L.)

* Author to whom correspondence should be addressed; E-Mail: barea@depeca.uah.es; Tel.: +34-918-856-574; Fax: +34-918-856-591.

Received: 7 November 2010; in revised form: 19 December 2010 / Accepted: 20 December 2010 / Published: 29 December 2010

\begin{abstract}
This paper describes a sensory system for implementing a human-computer interface based on electrooculography. An acquisition system captures electrooculograms and transmits them via the ZigBee protocol. The data acquired are analysed in real time using a microcontroller-based platform running the Linux operating system. The continuous wavelet transform and neural network are used to process and analyse the signals to obtain highly reliable results in real time. To enhance system usability, the graphical interface is projected onto special eyewear, which is also used to position the signal-capturing electrodes.
\end{abstract}

Keywords: electrooculography; eye movement; human-computer interface; wavelet transform, neural network

\section{Introduction}

Much research is under way into means of enabling the disabled to communicate effectively with a computer [1,2], as development of such means has the potential to enhance their quality of life considerably. Depending on users' capabilities, systems such as speech recognition, brain-computer interfaces [3], and infrared head-operated joysticks [4], etc. may be employed for this purpose. 
For users with sufficient control of their eye movements, one option is to employ a gaze-direction detection system to codify and interpret user messages. Eye-movement detection interfaces may be based on videooculography (VOG) [5], infrared oculography (IORG) [6] and electrooculography (EOG). Furthermore, this type of interface need not be limited to severely disabled persons and could be extended to any individual with sufficient eye-movement control.

EOG is a widely and successfully implemented technique and has proven reliable and easy to use in human-computer interfaces (HCI). Gips et al. present an electrode-based device designed to enable people with special needs to control a computer with their eyes [7]. Barea described an HCI based on electrooculography for assisted mobility applications [8]. The paper studies the problems associated with using EOG to control graphical interfaces and proposes an electrooculographic ocular model to resolve these issues. It also discusses graphical interfaces' various access options (direct, scanning, gestures, etc.). Zheng et al. describe an eye movement-controlled HCI designed to enable disabled users with motor paralysis and impaired speech to operate multiple applications (such as communication aids and home automation applications) [9]. Ohya et al. present development of an input operation for the amyotrophic lateral sclerosis communication tool utilizing EOG [10]. Bulling et al. describe eye-movement analysis for activity recognition using electrooculography [11]. EOG-based systems have also been developed in the robotics field to control mobile robots [12,13] and guide wheelchairs $[14,15]$.

Various techniques may be used to model the ocular motor system using EOG and detect eye movements. These include saccadic eye-movement quantification [16], pattern recognition [17], spectral analysis [18], peak detection deterministic finite automata [19], multiple feature classification [20], the Kalman filter [21], neural networks [22-25] and the support vector machine [26]. Notable efforts have also been made to reduce and eliminate the problems associated with gaze detection in EOG, such as drift, blink, overshoot, ripple and jitter [12,27].

Recent new research has focused on using electrooculograms to create efficient HCIs [18,28,29] and developing novel electrode configurations to produce wearable EOG recording systems, such as wearable headphone-type gaze detectors [21], wearable EOG goggles [17,30,31], or light-weight head caps [32].

The electrooculographic biopotential value varies from 50 to $3,500 \mu \mathrm{V}$ with a frequency range of about DC-100 Hz. This signal is usually contaminated by other biopotentials, as well as by artefacts produced by other factors such as the positioning of the electrodes, skin-electrode contact, head and facial movements, lighting conditions, blinking, etc. To minimize these effects, the system requires high-quality signal acquisition hardware, and suitable analysis algorithms need to be applied to the signal. Signal processing is usually performed on a personal computer. However, a more economical option, and one that also consumes less electricity, is to use a microcontroller-based system.

The purpose of this research paper is to develop a system to capture and analyse EOG signals in order to implement an HCI, as shown in Figure 1. The system comprises two electronic modules - the signal Acquisition Module (AM) and the Processing Module (PM). Eyewear incorporating a set of appropriately positioned dry electrodes captures the EOG signals, which the AM acquires, digitizes and transmits using the ZigBee protocol. 
Figure 1. System architecture.

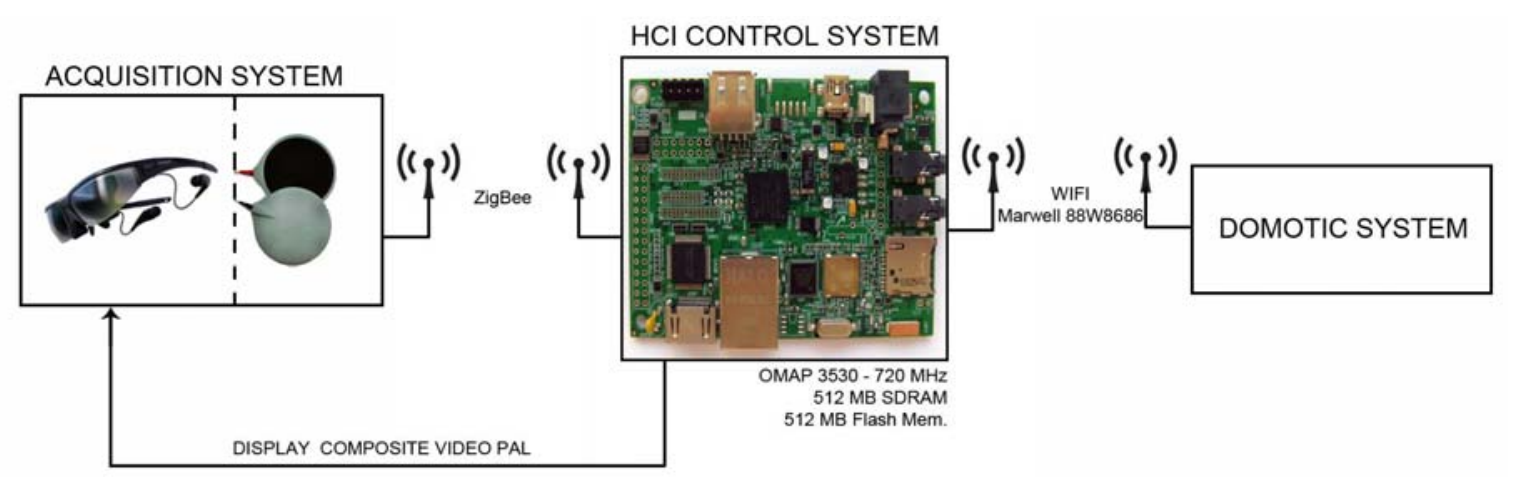

The PM receives the signals from the AM and executes the algorithms to detect the direction of the user's gaze. Simultaneously, it projects the user interface onto the eyewear and, according to the selection made by the user, transmits the commands via WiFi to a home automation system or performs other tasks (i.e., call a nurse, etc.).

This paper comprises seven sections. Section 2 describes the signal acquisition and ZigBee-enabled transmission circuit (AM), Section 3 describes the PM, Section 4 describes signal processing, and Sections 5, 6 and 7 present the results, discussion and conclusions of this paper.

\section{Acquisition System}

\subsection{Wearable EOG Goggles}

The eyewear, which is based on a commercially available model (Vuzix Wrap 230) [33] and features integrated electrodes, performs two functions - it holds the dry electrodes used to capture the EOG signal in position and serves as the medium onto which the user interface is projected. The electrooculogram is captured by five electrodes placed around the eyes. The EOG signals are obtained by placing two electrodes to the right and left of the outer canthi (A-B) to detect horizontal movement and another pair above and below the left eye (C-D) to detect vertical movement. A reference electrode is placed above the right eye (E). The eyewear has a composite video input (PAL format) and displays high-colour, high-contrast images at $320 \times 240$ resolution, equivalent to a 46 -inch screen viewed at a distance of 3 metres. Figure 2 shows the placement of the electrodes in the eyewear.

Figure 2. EOG goggles based on Vuzix Wrap 230 eyewear.
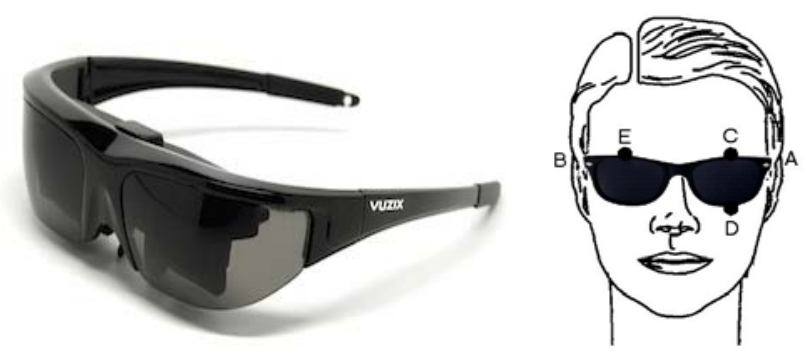


\subsection{Acquisition System}

The EOG signal is influenced by several factors, including eyeball rotation and movement, eyelid movement, and various artefact sources (electrode placement, head and facial movement, lighting, etc.). As the shifting resting potential (mean value) changes, it is necessary to eliminate this value. To do so, an AC high-gain differential amplifier $(1,000-5,000)$ is used, together with a high-pass filter (0.05-Hz cut-off frequency), a relatively long time constant, and a low-pass filter (35-Hz cut-off frequency). The signals are sampled 100 times per second.

A two-channel amplifier has been designed and developed to capture bioelectric signals and transmit them using the ZigBee protocol (wireless). This small, portable system's power supply has been optimized to enable battery-powered operation. One of its main advantages is its versatility, since it enables each channel to be configured dynamically and individually (active channel adjustment, channel offset, gains, sampling frequency or driven-right-leg circuit gain) via commands sent over the ZigBee protocol. Figure 3 shows the electrical system diagram for data capture, amplification, digitization and transmission.

Figure 3. Electrical system diagram.

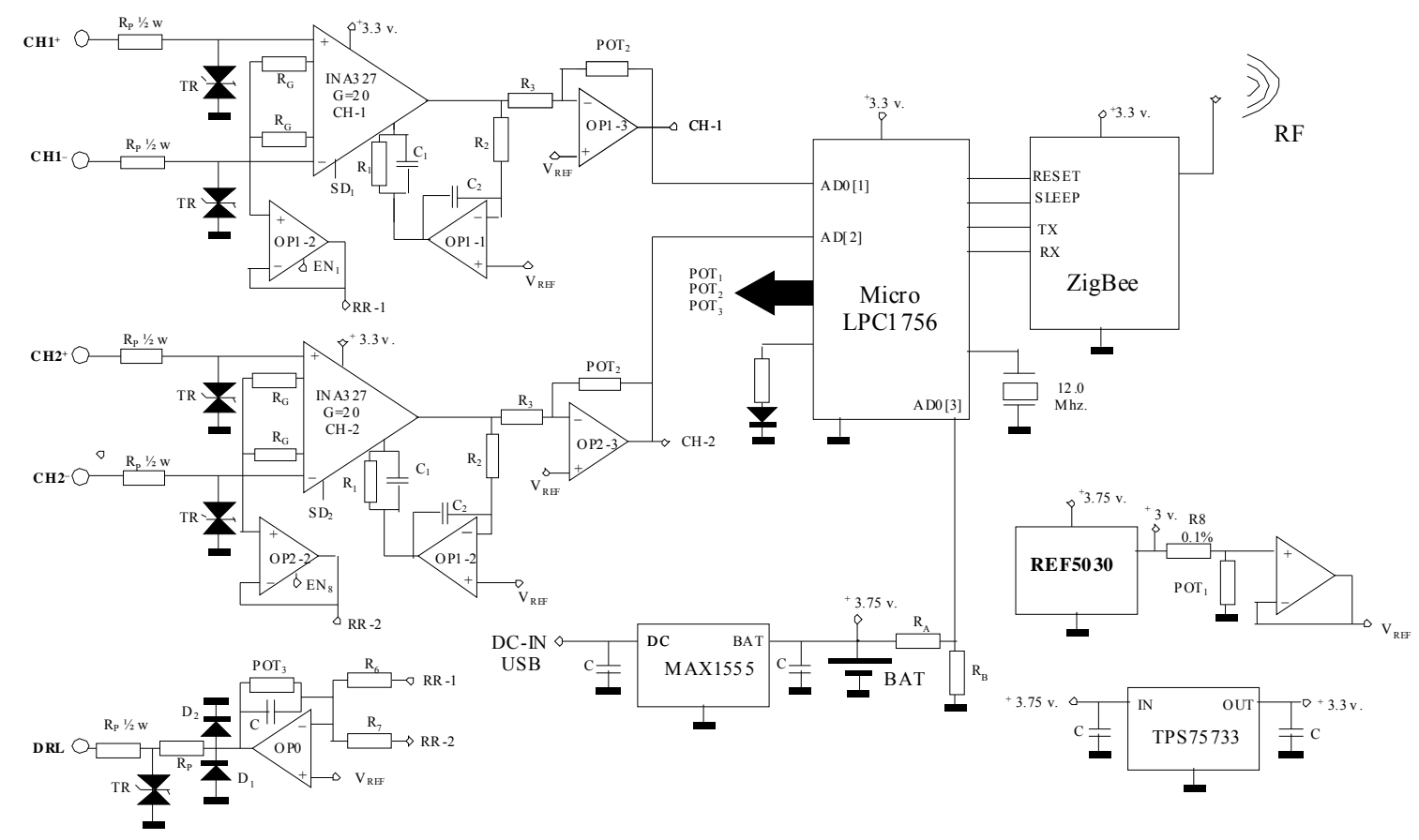

The analogue signal acquisition hardware comprises two differential inputs $(\mathrm{CH} 1, \mathrm{CH} 2)$, which are digitized by the microcontroller's internal ADC (LPC1756, 12-bit resolution, 100-300 Hz sampling frequency adjustable in 10-Hz steps) before being transmitted via the ZigBee protocol.

The system communicates via the ZigBee protocol, acting directly on the link level (802.15.4). Due to its low energy consumption and widespread implementation in low-cost commercial systems, this protocol is considered the best option. The XBee module is connected to the microcontroller by $\mathrm{D}_{\mathrm{OUT}}$ and $\mathrm{D}_{\mathrm{IN}}$ lines. The XBee module and the microcontroller communicate via an 115,200 bps serial connection and the XBee module is controlled by API frames. The XBee device's command set allows 
users to configure the network and serial interface parameters via the microcontroller. Although this paper considers ZigBee the best option because of its low energy consumption, the electrical system diagram can be easily modified to implement Bluetooth, which is a much more widely used communications standard, although it requires considerably more power.

The device is powered by rechargeable lithium-ion batteries (3.75 V DC/6.8 Ah). Consumption has been reduced by using integrated circuits with a shutdown (SD) feature, which means they can be deactivated when not needed by the active application. The batteries are recharged from a computer USB port using a MAX1555 integrated circuit.

The integrated circuits have a $3.3-\mathrm{V}$ power supply provided by regulator TPS75733, which draws power directly from the battery. The circuit uses a very stable 3.0-V reference voltage (REF5030). A digital potentiometer $\left(\mathrm{POT}_{1}: \mathrm{MCP} 4261\right)$ is then used to transform this into a variable reference voltage $\left(\mathrm{V}_{\mathrm{REF}}\right)$. This potentiometer is adjusted via the microcontroller using the serial peripheral interface (SPI) protocol and is used to configure each channel.

The amplification margin of the signals recorded by the two channels is established independently (0-5,000 adjustable gain). The lower cut-off frequency is set at $0.05 \mathrm{~Hz}$ and the upper cut-off frequency is set at $35 \mathrm{~Hz}$.

One of the aspects bearing most heavily on final system quality is front-end amplification of the bioelectrical signal. In many cases, the bioelectrical signals' amplitudes are below $50 \mu \mathrm{V}$ and are usually contaminated by various noise sources, such as the network alternate component (50 or $60 \mathrm{~Hz}$ and its harmonics), electrode contact noise (baseline drift), other physiological patient systems (i.e., muscular noise), interference from electronic devices, etc. To minimize these effects, various techniques may be used to optimize analogue signal capture. The proposed architecture employs two-stage signal amplification. The first stage comprises a differential amplifier $\left(G_{1}=20=R_{1} / R_{G}\right)$ based on an instrumentation amplifier (INA327) with a shutdown feature $\left(\mathrm{SD}_{\mathrm{i}}\right)$, which enables unused channels to be deactivated. This first stage's general gain expression is shown below:

$$
G_{1}(s)=\frac{R_{1} / R_{G}}{1+s \cdot R_{1} \cdot C_{1}} \cdot \frac{s \cdot C_{2} \cdot R_{2}}{1+s \cdot C_{2} \cdot R_{2}}
$$

This amplifier has a mean frequency gain of $\mathrm{R}_{1} / \mathrm{R}_{\mathrm{G}}$, a lower cut-off frequency of $0.05 \mathrm{~Hz}$ $\left(2 \pi R_{2} \cdot C_{2}\right)^{-1}$ and an upper cut-off frequency of $\left(2 \pi R_{1} \cdot C_{1}\right)^{-1}$. The upper cut-off frequency of the input stage is set by the $R_{1} \cdot C_{1}$ product. As $R_{1}$ should be kept constant to avoid modifying the amplifier gain, $\mathrm{C}_{1}$ has been modified to produce an upper cut-off frequency of $35 \mathrm{~Hz}$.

The second amplification stage uses the OPA2334 operational amplifier, which has been chosen for its low offset voltage. As it is an adjusted gain inverting amplifier $\left(\mathrm{G}_{2}=-\mathrm{POT}_{2} / \mathrm{R}_{3}\right)$, the gain can be set between $0\left(\mathrm{POT}_{2}\right.$ resistance $\left.=0 \Omega\right)$ and -250 (256 steps), while signal amplification allows total gain per channel of $0-5,000$.

The Driven-Right-Leg (DRL) circuit allows common mode signal reduction, applying a circuit feedback voltage to the patient [34]. Each channel's common mode signal is captured by a voltage follower (OPi-2 = OPA2334) and another similar amplifier adds them together. The feedback circuit gain can be adjusted by commands from the host using $\mathrm{POT}_{3}$ (MCP4261) on each acquisition channel. This improves signal capture, as the patient's potential value depends on many factors (electrode 
location, proximity to the feedback network, stretcher type, etc.). Figure 4 shows the implemented AM.

Figure 4. Image of the AM.

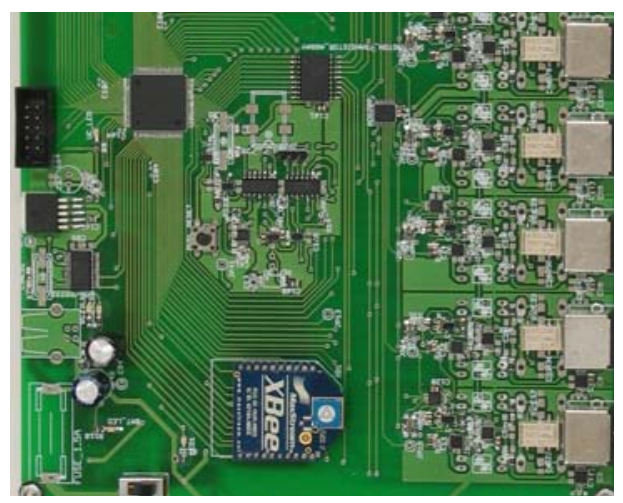

\section{Processing Module}

The PM's function is to receive the EOG signals via the ZigBee protocol, apply the appropriate algorithms to detect the user's eye movements, display the user interface on the eyewear, decodify the user's message, and send the appropriate command via WiFi to the home automation system that will execute the user's instructions (switch on TV, etc.). In addition, during the training and calibration phases, the PM captures the user's eye movements and with them trains a radial-basis-function (RBF) neural network using the Extreme Learning Machine (ELM) algorithm. It then sends the commands to the AM to adjust the system's operating parameters (amplifier gain, offset, etc.).

The PM is based on a high-performance SoC (System on Chip), the OMAP3530, which includes a Cortex-A8 core as well as a C64x + DSP running at $720 \mathrm{MHz}$. It has $512 \mathrm{MB}$ of RAM and $512 \mathrm{MB}$ of flash memory. It provides a direct composite video output (compatible with the PAL and NTSC formats) connected to the Vuzix Wrap 230 eyewear.

The operating system used by the processing card is an OpenEmbedded-based Linux distribution optimized for the ARMv7 architecture with the ARM/Linux kernel (version 2.6.32) and the U-Boot 1.3.4 bootloader. The OpenEmbedded-based file system includes the XFCE-lite graphic environment. Using a Linux environment provides access to a multitude of graphic and console applications and utilities. The system has the capacity to compile its own programs as it includes the GCC compiler and auxiliary native tools (Binutils).

The PM communicates with the AM via a ZigBee link. To achieve this, two commercial ZigBee modules (XBee) are connected to the respective UARTs on the processing (OMAP3530) and acquisition (LPC1756) cards' microcontrollers. Communications via the ZigBee protocol are performed with a power level of $0 \mathrm{dBm}$.

Data acquisition via the ZigBee protocol is performed constantly and in real time. To achieve this, a high-priority process scans the buffer of the UART connected to the ZigBee module and sends these data to the two processes responsible for executing the signal-processing algorithms (wavelet transform and neural network).

Once the user's eye movements in relation to the user interface projected onto the eyewear have been decodified and interpreted, the orders are sent via WiFi (because of its universality) to a home 
automation system or similar application. The WiFi interface is implemented using a Marvell $88 \mathrm{~W} 8686$ (IEEE $802.11 \mathrm{~b} / \mathrm{g}$ ) chipset connected to the OMAP3530. The WiFi stack is part of the ARM/Linux 2.6 kernel (Linux wireless subsystem, IEEE-802.11) and includes the necessary wireless tools (iwconfig, iwlist, etc).

\section{Signal Processing}

Figure 5 shows the processing performed on the digitized EOG signal. Processing is structured into two phases. The first phase is optional and consists of adjusting the signal-capture system, applying the linear saccadic eye model and training the neural network according to the user's signals. This option can be activated when a new user utilizes the system or when the user's responses change due to tiredness or loss of concentration. Parameter adjustment should be performed by someone other than the user. The acquisition module allows for adjustment of channel gain, DRL gain and the $V_{\text {REF }}$ parameter. The ocular model calculates the relationship between EOG variation and the eye movement performed, as well as calculating the minimum detection threshold.

Figure 5. Saccadic eye-movement detection process.

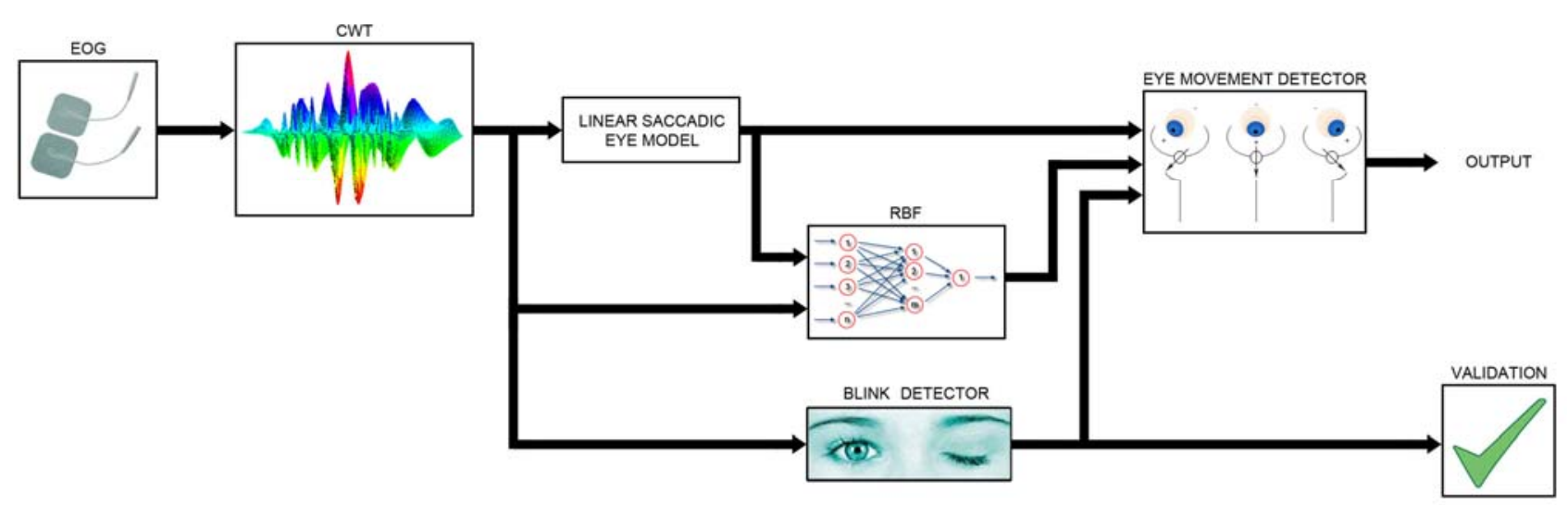

Once appropriate signal-capture conditions are established, the system instructs the user to look at a series of pre-determined positions on the user interface. The EOG signal is filtered by the wavelet transform and a linear saccadic model is used to detect and quantify saccadic movements. These signals are then used as samples to train the neural network. The neural network's purpose is to enhance detection of saccadic movements by using pattern recognition techniques to differentiate between variations in the EOG attributable to saccadic movements and those attributable to fixation problems or other artefacts. As users become tired and their concentration deteriorates (particularly after long periods of operation), these artefacts in the EOG signal become increasingly pronounced.

The continuous wavelet transform (CWT) is useful for detecting, characterizing and classifying signals with singular spectral characteristics, transitory content and other properties related to a lack of stationarity [35]. In the case addressed here, the best results were obtained by using the db1 mother wavelet from the Daubechies family due to its strong correlation with the changes the system aims to detect in the original EOG signal. The CWT makes use of modulated windows of variable size adjusted to the oscillation frequency (i.e., the window's domain contains the same number of 
oscillations). For this reason, the method employs a single modulated window, from which the wavelet family is obtained by dilation or compression:

$$
\Psi_{b, a}(t) \rightarrow \frac{1}{\sqrt{|a|}} \Psi\left(\frac{t-b}{a}\right)
$$

where $a \neq 0$ and $\mathrm{b}$ are the scale and latency parameters, respectively. The energy of the functions is preserved by a normalized factor $1 / \sqrt{|a|}$.

The optimal scale that produced greatest correlation in the studies carried out was $a=60$. The effect of this wavelet is similar to that of deriving the signal (high-pass filtering), although the results are magnified and it is easier to identify saccadic eye movements as the threshold is not as critical.

The linear saccadic model considers that the behaviour of the EOG is linear. This is equivalent to stating that the eye movement is a constant of the variation of the EOG (eye movement $=\mathrm{k}^{*} \mathrm{EOG}$ _variation) [8]. A saccadic movement is considered to occur when the EOG derivative exceeds the minimum threshold. The direction and size of a saccade is given by its sign and amplitude. The neural network implemented is a radial-basis-function (RBF) network trained using the ELM algorithm [36], which is characterized by its short computational time. The network's input data comprise the contents of a 50 -sample time window ( 25 preceding samples and 25 subsequent samples) from the EOG signal corresponding to a detected eye movement and are processed using the wavelet transform. The internal structure has 20 neurons in the hidden layer. The network's output determines whether a valid saccadic movement has occurred. Network training is performed on a set of 50-sample segments taken from the EOG at different instants. These correspond to resting (gaze directed at the centre), saccadic eye movements, and fixation periods. Output is "1" when a saccadic movement exists and " 0 " in all other cases. The output of the neural network is a linear combination of the basis functions:

$$
f(x)=\sum_{i=1}^{N_{H}} \beta_{i} \cdot \exp \left(-\frac{\left\|w_{i} \cdot x-\mu_{i}\right\|^{2}}{\sigma_{i}^{2}}\right)=\sum_{i=1}^{N_{H}} \beta_{i} \cdot g_{i}(x)
$$

where $\beta_{\mathrm{i}}$ denotes the output weight matrix, $\mathrm{w}_{\mathrm{i}}$ are the input weights and $\sigma_{\mathrm{i}}$ is the width of the basis function.

The ELM algorithm is a learning algorithm for single hidden-layer feed-forward networks. The input weights $\left(\mathrm{w}_{\mathrm{i}}\right)$, centres $\left(\mu_{\mathrm{i}}\right)$ and width of the basis function are randomly chosen and output weights $\left(\beta_{\mathrm{i}}\right)$ are analytically determined based on the Moore-Penrose generalized inverse of the hidden-layer output matrix. The algorithm is implemented easily and tends to produce a small training error. It also produces the smallest weights norm, performs well and is extremely fast [37].

A block has also been designed to work in a similar way to a mouse click to enable users to validate the desired commands. This block detects two or three consecutive blinks within a time interval configured according to the user's capabilities. Blink detection is based on pattern recognition techniques (a blink template is created from user blink segments). Blinks are detected by comparing the template against the EOG's vertical component. A blink is considered to exist when there is a high level of similarity (above a pre-determined threshold) between the template and the EOG's vertical component. Figure 6 shows an example of the difference on the vertical EOG between blinking and an 
upward saccadic movement. As may be seen in the EOG recorded in each case, the duration of the blink is shorter than that of the saccadic movement.

Figure 6. Effect of blinking on the vertical EOG.

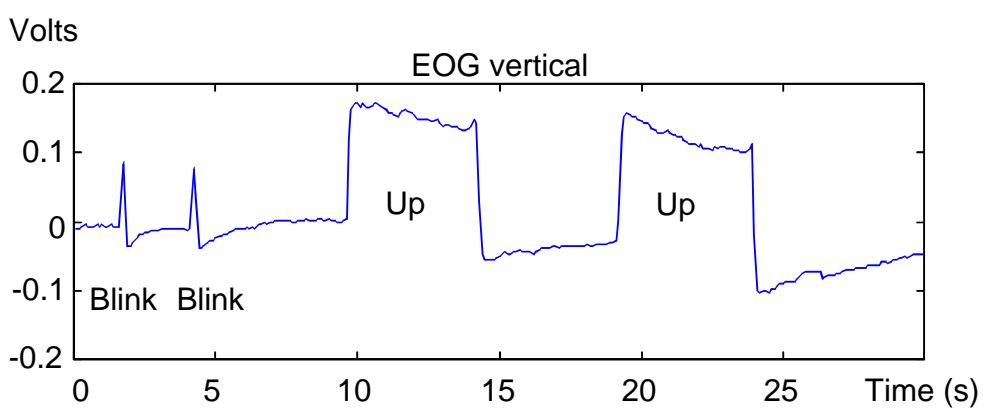

Finally, based on the neural network, linear saccadic model and blink detector outputs, the eye-movement detector block determines the validity of the saccadic movement detected. When the linear saccadic model detects a saccadic movement, a 50-sample window from the EOG signal (centred on the instant the saccadic movement is detected) is input into the neural network. The network output determines the movement's validity. Meanwhile, to eliminate the blink effect on the EOG signal, when a blink is detected, the saccadic movement detected at the same instant is discarded. Furthermore, as the neural network's training segment is longer than a blink segment, the effect can be filtered immediately by the neural network to remove false saccadic movements.

As regards the system's computational time, a $260.49 \mathrm{~ms}$ delay exists between performance of a saccadic movement and its validation. This delay may be considered appropriate for typical graphical interface control applications. Figure 7 shows a timeline displaying the various processing stage times. The EOG signals are sampled 100 times per second. $1.68 \mathrm{~ms}$ are needed to process the CWT, while the linear saccadic model takes $0.012 \mathrm{~ms}$ to detect the movement and quantify it. Blink detection takes $0.26 \mathrm{~ms}$. A $250 \mathrm{~ms}$ delay is needed after a saccadic movement is detected (corresponding to 25 subsequent samples from the EOG signal) before the signal can be propagated over the neural network (50 samples). Signal propagation over the RBF takes $8.52 \mathrm{~ms}$. Finally, the eye-movement detector block requires $0.035 \mathrm{~ms}$ to validate the movement performed.

Figure 7. Timeline.
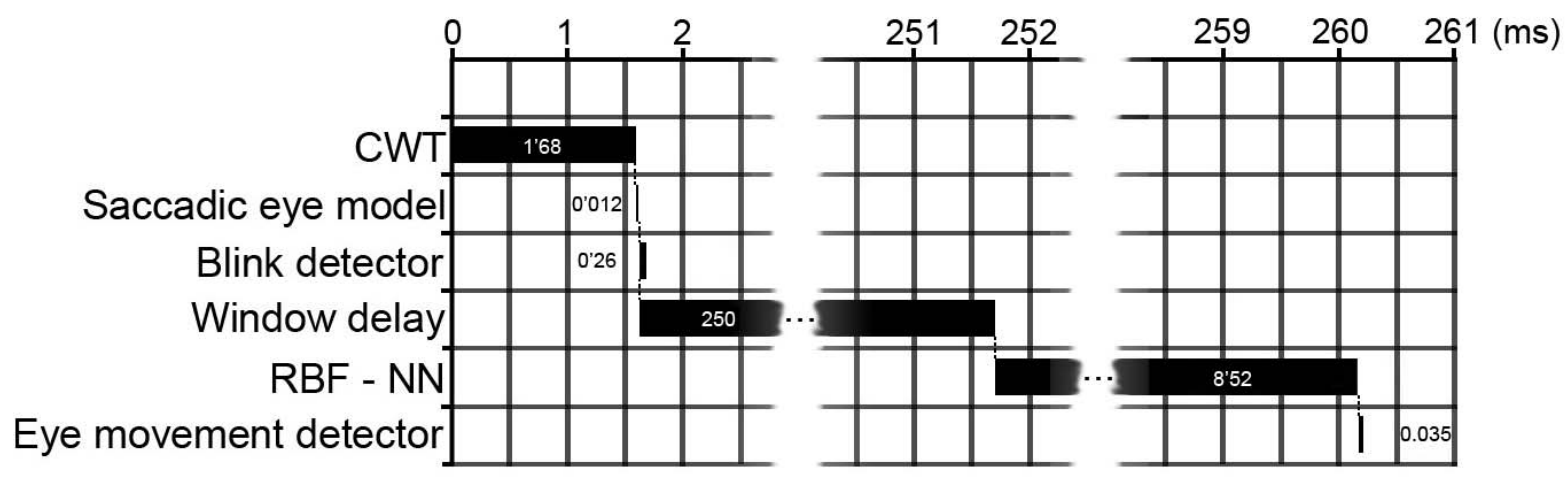
Although this may appear to be a long delay, it only occurs when a saccadic movement is detected. In all other cases, the signal is not propagated over the RBF and the system's computational time stands at $1.975 \mathrm{~ms}$. Given that the system acquires a sample every $10 \mathrm{~ms}$, to all practical intents and purposes it processes the EOG signal in real time. The second phase comprises a cyclical process in which the EOG is captured and the signals are processed to determine which command the user wishes to activate. Figure 8 shows an example of processing of a typical horizontal-channel signal-the user's gaze progressively shifts 10, 20, 30 and 40 degrees horizontally [Figure 8(a)]. First, the EOG signal is filtered using a CWT [Figure 8(b)], then the linear saccadic model detects saccades and determines their angle [Figure 8(c)]. When a movement is detected, the EOG signal is input into the neural network, which determines if it is an eye movement [Figure 8(d)]. Finally, based on the neural network and blink detector outputs, the eye-movement detector determines the validity of the saccadic movement. It then quantifies the movement according to the value obtained in the linear saccadic model [Figure $8(\mathrm{e})]$.

Figure 8. Eye-movement detection process sequence.

(a)

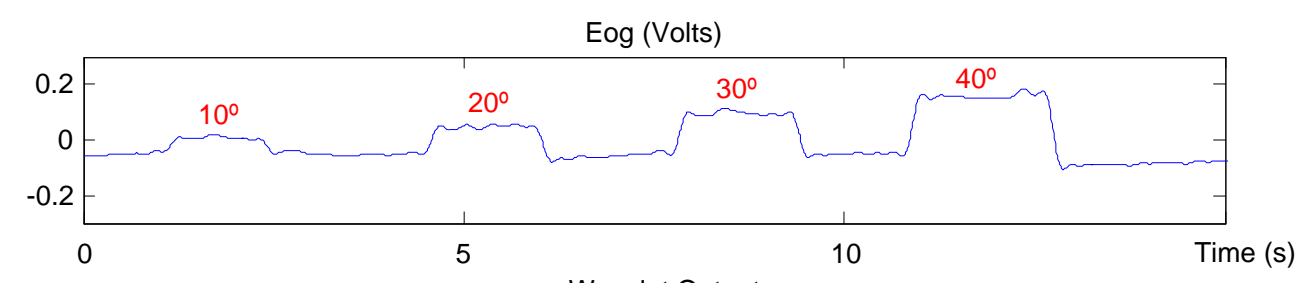

(b)

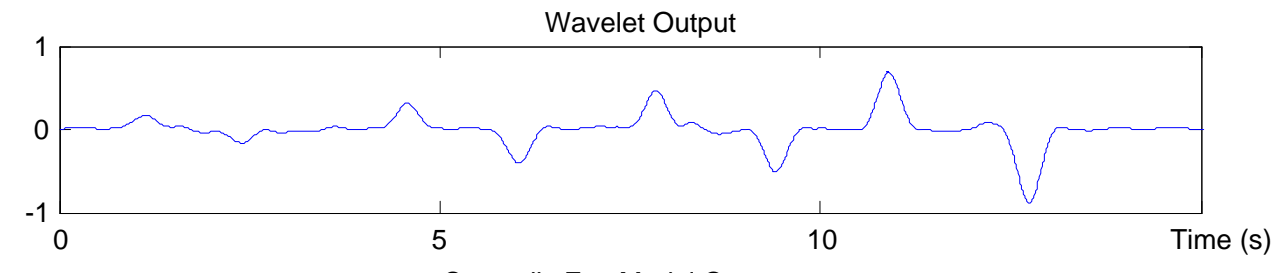

(c)

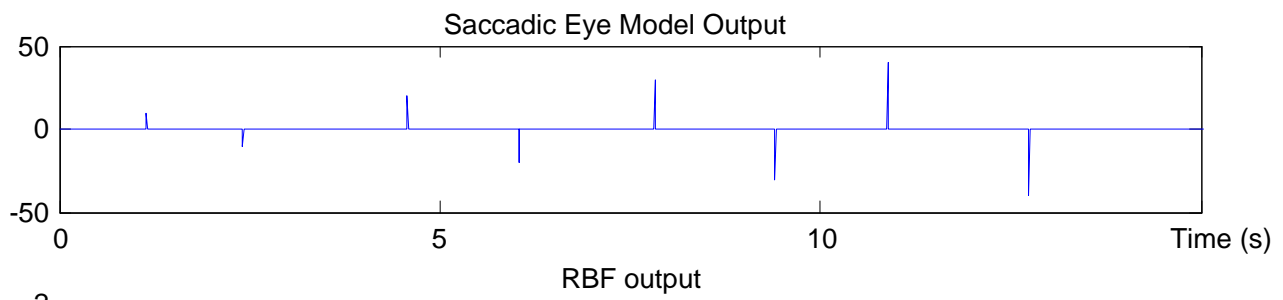

(d)

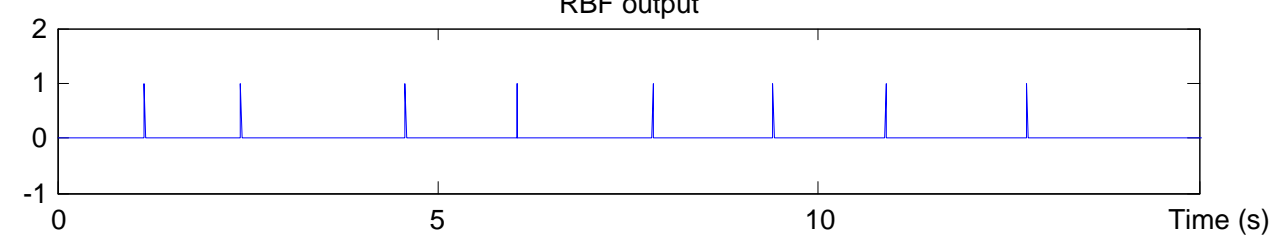

(e)

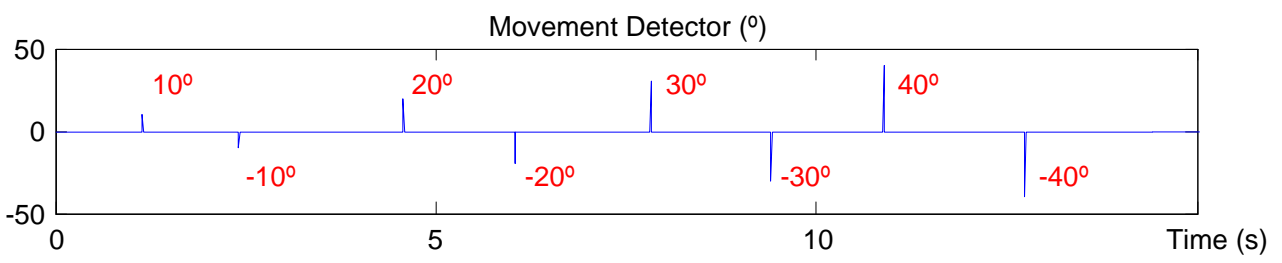

The system developed is able to detect eye movements to within an error of 2 degrees, making it possible to select or codify a large number of commands within a particular graphical interface. 
Furthermore, the validation block makes it possible to validate the command selected or eye movement performed.

\section{Results}

This paper implements a prototype wearable HCI system based on electrooculography. The eyewear is used to position the electrodes and display the user interface, thereby facilitating system usability. Signal capture is performed by a low-power-consumption electronic circuit. The prototype's intelligent core is based on a high-performance microcontroller that analyses the signals and transmits the user's commands to a home automation system via a WiFi connection.

Eye movement-based techniques employed to control HCIs include Direct Access, Scanning and Eye Commands (gestures). Direct Access is the most widely used form (in which the user, when shown a graphical interface, selects the desired command by positioning a cursor over it and then carrying out a given validation action, usually a mouse click). If the graphical interface is vision-controlled, the cursor is directed by eye movements and validation is performed either on a time basis or by an ocular action such as blinking. The drawback of this interface is the 'Midas Touch' problem, as the human eye is always active. Therefore, it is necessary to ensure that validation cannot be performed involuntarily. To avoid this problem, eye-movement codification is generally used. The aim of this technique is to develop control strategies based on certain eye movements (ocular actions or gestures) and their interpretation as commands. Usually, eye-movement recognition is based on detecting consecutive saccades, which are then mapped to eye movements in basic directions-left, right, up and down [18,19,29,38].

As quality in graphical interface control is partly measured in terms of ease-of-use and system simplicity, the Direct Access technique was selected as it is the most natural and fastest and, therefore, the most comfortable to use. Furthermore, it also allows the system to include a large number of commands without the need for users to memorize complex ocular actions.

To operate the system, the user looks at the centre of the screen and then looks at the desired command (saccadic movement). This selects the command, which is then validated by two consecutive blinks within a pre-determined time limit, which starts when the eye movement commences and is configured according to the user's capabilities.

As the system detects the eye-movement angle to within an accuracy of 2 degrees, it is technically possible to design an interface containing a large number of commands. However, experience shows that a simpler interface with 4-8 commands is preferable, as the capabilities of users likely to operate these interfaces need to be taken into account. This means detecting simple up, down, left and right movements and their corresponding diagonals. Figure 9 shows one of the interfaces implemented.

The system was tested by five volunteers (three men and two women) aged between 22 and 40 using an 8-command user interface. Thirteen 5-minute tests were performed per volunteer (1 hour in total). The tests required volunteers to select each of the interface's commands cyclically $(13 \times 5 \times 8=520$ selections in total, 104 per volunteer). Once a saccadic movement was detected, the user had 2 seconds to perform validation (double blink). 
Figure 9. Eight-command interface.

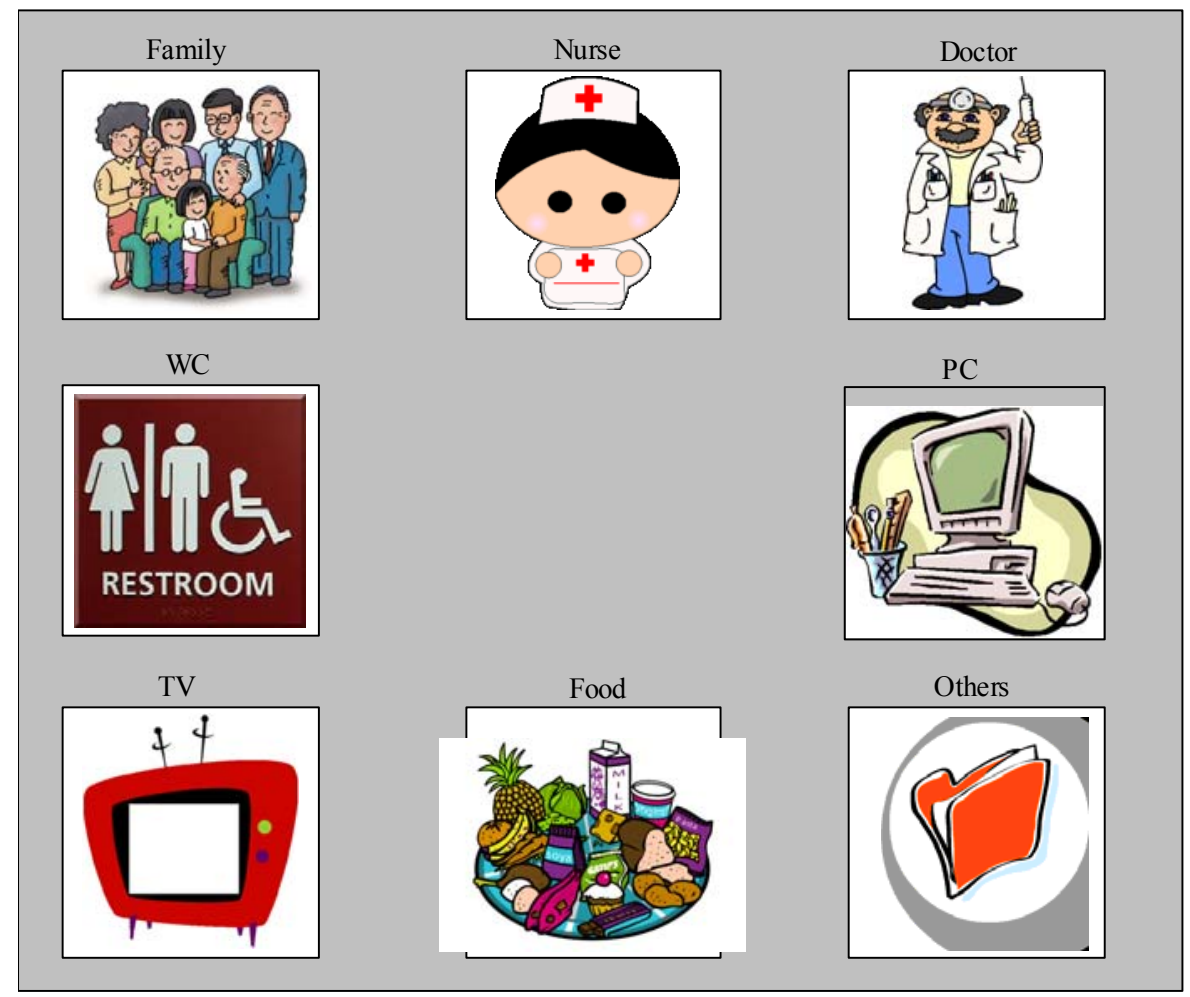

Training for volunteers to familiarize themselves with the system took approximately 5 minutes and during this time a member of the research group calibrated the system (gain, offset, etc.) for each volunteer. The system then trained the neural network using the real data captured from each volunteer.

Table 1. Experiment results.

\begin{tabular}{lcc}
\hline & Successes & Failures \\
\hline User 1-man & 89 & 15 \\
User 2-woman & 98 & 6 \\
User 3-man & 94 & 10 \\
User 4-woman & 100 & 4 \\
User 5-man & 97 & 7 \\
\hline
\end{tabular}

As table 1 shows, the volunteers achieved an overall success rate of $92 \%$. The errors produced were due to problems in either saccadic movement detection ( $66 \%$ of errors) or command validation (34\% of errors). Figure 10 shows the distribution of these failures by test.

The following may be concluded from the results obtained:

- The number of failures is low initially because user concentration is high. Providing prior training improved these results, as the user was already accustomed to operating the HCI and, furthermore, the neural network was trained on each user's own signals. 
- The number of failures increases with time, a trend principally attributable to falling user concentration and increasing tiredness. However, the number of failures is much lower than when using other electrooculographic models [8].

Figure 10. HCI errors in relation to time.

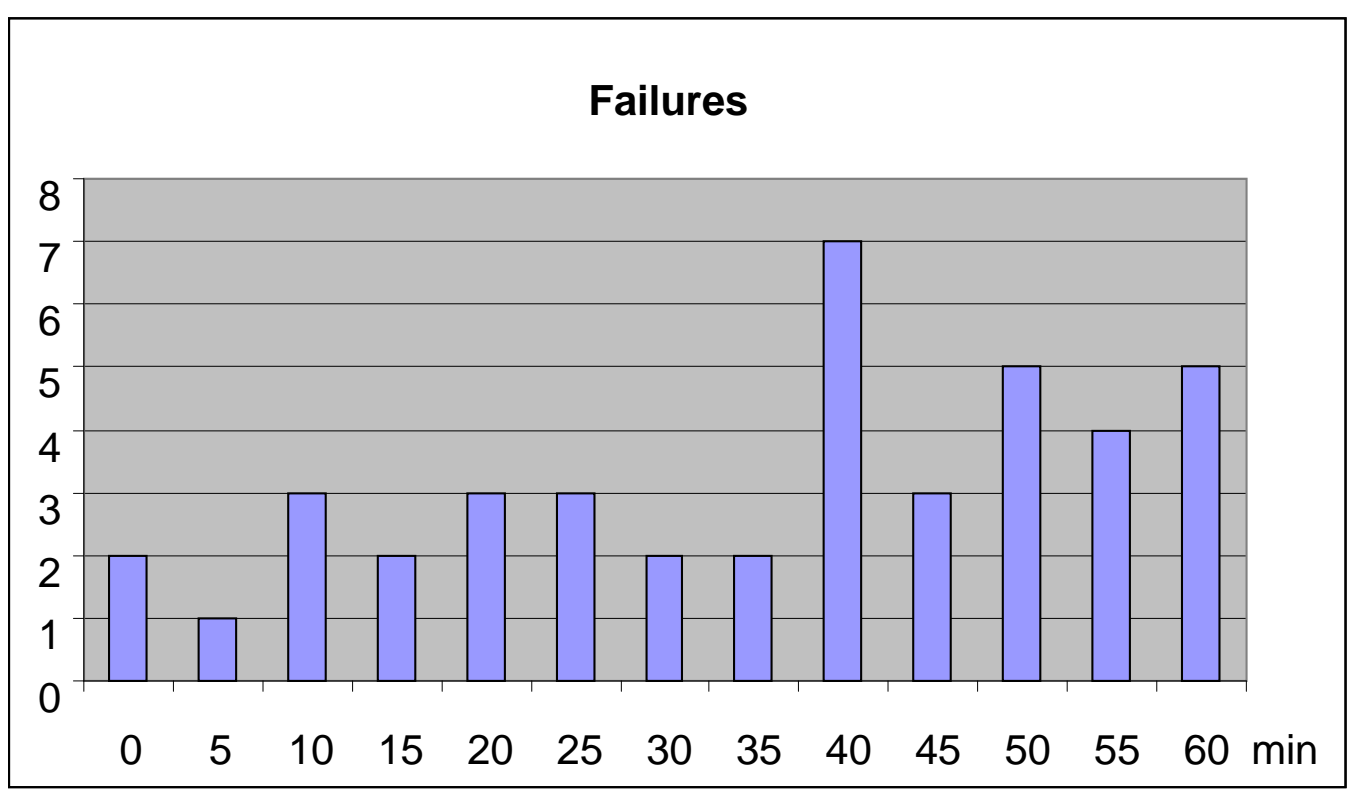

These results, which naturally may vary according to users' physical and mental capabilities, preliminarily demonstrate that the system implemented operates as intended.

\section{Discussion}

Much of recent research into EOG-based HCI systems focuses on (a) developing wearable systems, and (b) enhancing system reliability by implementing new processing algorithms. This paper presents advances in both regards. On the one hand, it implements a modular hardware system featuring wearable goggles and a processing unit based on a high-performance microcontroller and, on the other, the EOG signal-processing technique employed provides satisfactory HCI control.

This paper uses a continuous wavelet transform to filter the EOG signal and employs a neural network to provide robust saccadic-movement detection and validation. The system has been validated by 5 healthy users operating a Direct Access eight-command HCI.

Initial EOG signal processing using the continuous wavelet transform enhances the non-stationary and time-varying EOG signal [39] and therefore, in our case, makes identification of smaller saccadic movements possible. This is vitally important when working with Direct Access interfaces that require highly accurate eye-movement detection. In this paper, the best results were obtained using the db1 mother wavelet from the Daubechies family at scale 60. Other papers have employed the continuous 1-D wavelet coefficients from the signal at scale 20 using the Haar wavelet [30]. This paper's authors obtained better results with the Daubechies mother wavelet than with the Haar mother wavelet or with conventional or adaptive filtering techniques (Wiener filter, etc.) [8].

Neural networks have long been used successfully to process EOG signals [22,23]. Various training algorithms and architectures have been researched and have produced generally satisfactory 
results $[24,40,41]$. Both the neural network architecture used in this case (RBF), and its training algorithm (ELM), were optimized for real-time use on a microcontroller-based system.

As commented in the Results section, eye movement-based techniques employed to control HCIs include Direct Access, Scanning and Eye Commands (gestures). Direct Access is the most widely implemented technique because it is the most natural and the fastest and, therefore, the most comfortable to use. Furthermore, it also allows the system to include a large number of commands without the need for users to memorize complex ocular actions. Drawbacks associated with this interface include the 'Midas Touch', eye jitter, multiple fixations on a single object, etc. To avoid these problems, one widely used option is to develop applications based on eye-movement codification or gestures $[16,42]$. The system presented in this paper accurately detects all eye movements, which means that the resulting gesture-based HCI (using codified up, down, right and left eye movements) is extremely robust.

As yet, a testbed widely accepted by researchers to measure and compare the results of EOG-based HCI systems does not exist. User numbers and characteristics, user interfaces and experiment length, among other aspects, all vary from paper to paper. In this paper, the tests designed to generate messages valid for a home-automation system performed by five healthy volunteers produced an overall $92 \%$ success rate. The authors consider this sufficient to ensure satisfactory user communication. These results are similar to those achieved in other recent papers describing development of applications based on eye-movement codification. Nevertheless, most of these papers show the results obtained when detecting up, down, left and right eye movements, which are significantly easier to detect than other types of eye movement. For example, in a work by Deng et al., $90 \%$ detection accuracy is obtained for these movements and the system is used to control various applications/games [38]. In Gandhi et al. [19], detection and device-control accuracy is 95.33\%. The nearest neighbourhood algorithm is used by Usakli et al. to classify the signals, and classification accuracy stands at 95\% [28]. In a work by Bulling et al., eye movements are studied to detect gestures used to control a graphical interface. Accuracy (around 90\%) is calculated as the ratio of eye movements resulting in a correct gesture to the total number of eye movements performed [43].

However, few papers on EOG quantify movement detection accuracy and those that do quantify it do not employ the same parameters, thereby preventing exhaustive comparison between them. In this paper, the combination of the wavelet transform, the ocular model and the neural network produce a measurement error of less than 2 degrees. This error is in the same order of magnitude as that of other EOG-based HCI systems [44,45]. Other authors, such as Manabe et al., report that the average estimation error is 4.4 degrees on the horizontal plane and 8.3 degrees on the vertical plane [21].

One of the contributions made by this paper is that the neural network eliminates or minimizes the fixation problems that appear when the user becomes tired and that become increasingly significant when the HCI is used for long periods. Comparison between the number of false saccadic movements detected in 60-minute EOG recordings by the linear saccadic model based on derivatives implemented in Barea et al. [16] and the architecture proposed in this paper demonstrates that saccadic-movement detection errors due to fixation problems and artefacts derived from blinking have been practically eliminated. It is also noteworthy that although the error obtained in 20, 30 and 40-degree movements is in the order of 2 degrees (similar to that obtained in Barea [8]), a substantial improvement has been produced in detection of small saccadic movements (the error produced in detection of 10-degree 
saccadic movements has been reduced by $50 \%$ ). This is principally due to improvement of the $\mathrm{S} / \mathrm{N}$ ratio by the wavelet in comparison with conventional filtering techniques.

As regards the system's computational time, this stands at $260.49 \mathrm{~ms}$ when a saccadic movement is detected. Command validation time should also be added to this delay. In the system implemented in this paper, the principal bottleneck lies in the neural network, which requires a 500-ms EOG signal window. However, the improvement in result quality and reliability justifies neural network use. Furthermore, in most graphical interface control applications, this delay is not critical and does not affect usage of the system proposed. It should also be underlined that computational time when a saccadic movement is not detected stands at $1.975 \mathrm{~ms}$, which means that to all practical intents and purposes the system works in real time.

The authors propose the following areas for future research:

- System validation by a greater number of users (principally disabled users).

- Study of system performance in mobile settings. Although the results presented in this paper were obtained under static conditions, previous papers have examined conditions in which users were mobile [14]. This paper has developed algorithms to eliminate artefacts generated principally by errors deriving from electrode contact with the user's skin (skin-electrode interface) and facial movements or gestures. However, use of a different electrode type (dry electrodes) and a new method of attaching the electrodes to the user's face, as well as use of these systems in mobile settings, require in-depth study of the new problems/artefacts that may arise.

- Improvements to system features. On-line self-calibration of the ocular model parameters every time a new saccadic movement is detected. This would enable users to work with the model for long periods without the need for third-party intervention to calibrate the system if adjustment errors were detected.

- On-line neural network training. One of the advantages of using the ELM algorithm is its speed. In the tests performed in this paper, $14.5 \mathrm{~ms}$ were needed to train one hundred 50 -sample EOG segments. The short training time required makes it possible to perform on-line training every time a new saccadic movement is detected.

\section{Conclusions}

This paper presents a system to capture and analyse EOG signals in order to implement an HCI interface. Specific hardware has been developed to capture users' biopotentials and a Linux platform has been used to implement the algorithms and graphical user interface. The eyewear employed performs the dual function of capturing the EOG signal comfortably and implementing the user interface.

The results (92\% reliability) demonstrate that the system proposed works well and produces an error rate that permits its use as part of an HCI. As the system is portable, it may be easily implemented in home automation, robotic systems or other similar applications. Furthermore, the hardware platform's processing power provides scope to implement more complex signal-analysis algorithms. 


\section{Acknowledgements}

The authors would like to express their gratitude to the Ministerio de Educación (Spain) for its support through the project "Implementing a Pay as You Drive service using a hardware/software platform", ref. TSI2007-61970-C01.

\section{References}

1. Królak, A.; Strumiłło, P. Eye-blink controlled human-computer interface for the disabled. Adv. Soft Comput. 2009, 60, 123-133.

2. Usakli, A.B.; Gurkan, S.; Aloise, F.; Vecchiato, G.; Babiloni, F. On the use of electrooculogram for efficient human computer interfaces. Comput. Intell. Neurosci. 2010, doi:10.1155/2010/135629.

3. Wickelgen, I. Tapping the mind. Science 2003, 299, 496-499.

4. Evans, D.G.; Drew, R.; Blenkhorn, P. Controlling mouse pointer position using an infrared head-operated joystick. IEEE Trans. Rehabil. Eng. 2000, 8, 107-117.

5. Chan, S.; Chang, K.; Sang. W.; Won, S. A new method for accurate and fast measurement of 3D eye movements. Med. Eng. Phys. 2006, 28, 82-89.

6. Hori, J.; Sakano, K.; Saitoh, Y. Development of a communication support device controlled by eye movements and voluntary eye blink. IEICE Trans. Inf. Syst. 2006, 1790-1797.

7. Gips, J.; DiMattia, P.; Curran, F.X.; Olivieri, P. Using eagleeyes—an electrodes based device for controlling the computer with your eyes - to help people with special needs. In Proceedings of the 5th International Conference on Computers Helping People with Special Needs (ICCHP'96), Vienna, Austria, 1996; pp. 77-83.

8. Barea, R. HMI Based on Electrooculography. Assisted Mobility Application. Ph.D. Thesis, University of Alcalá, Alcalá, Spain, 2001.

9. Zheng, X.; Li, X.; Liu, J.; Chen, W.; Hao, Y. A portable wireless eye movement-controlled Human-Computer interface for the disabled. In Proceedings of the International Conference on Complex Medical Engineering, Tempe, AZ, USA, April 2009; pp. 1-5.

10. Ohya, T.; Kawasumi, M. Development of an input operation for the amyotropic lateral sclerosis communication tool utilizing EOG. Trans. Jpn. J. Soc. Med. Biol. Eng. 2005, 43, 172-178.

11. Bulling, A.; Ward, J.A.; Gellersen, H.; Tröster, G. Eye Movement Analysis for Activity Recognition Using Electrooculography. IEEE Trans. Pattern Anal. Mach. Intell. 30 March 2010.

12. Chen, Y.; Newman, W.S. A human-robot interface based on electrooculography. In Proceedings of the International Conference on Robotics and Automation (ICRA'04), New Orleans, LA, USA, April 2004; pp. 243-248.

13. Kim, Y.; Doh, N.L.; Youm, Y.; Chung W.K. Robust discrimination method of the electrooculogram signals for human-computer interaction controlling mobile robot. Intell. Autom. Soft Comp. 2007, 13, 319-336.

14. Barea, R.; Boquete, L.; Mazo M.; Lopez, E. System for assisted mobility using eye movements based on electrooculography. IEEE Trans. Neural. Syst. Rehabil. Eng. 2002, 4, 209-218. 
15. Yanco, H.A.; Gips, J. Drivers performance using single swicth scanning with a powered wheelchair: Robotic assisted control versus traditional control. In Proceedings of the Association for the Advancement of Rehabilitation Technology Annual Conference RESNA'98, Pittsburgh, PA, USA, 1998; pp. 298-300.

16. Barea, R.; Boquete, L.; Bergasa, L.M.; Lopez E.; Mazo, M. Electrooculograpic guidance of a wheelchair using eye movement's codification. Int. J. Rob. Res. 2003, 22, 641-652.

17. Brunner, S.; Hanke, S.; Wassertheuer, S.; Hochgatterer, A. EOG Pattern Recognition Trial for a Human Computer Interface. In Proceedings of 4th International Conference on Universal Access in Human-Computer Interaction, UAHCI 2007 Held as Part of HCI International 2007 Beijing, China, July 22-27, 2007; pp. 769-776.

18. Lv, Z.; Wua, X.; Li, M.; Zhang, D. A novel eye movement detection algorithm for EOG driven human computer interface. Pattern. Recognit. Lett. 2010, 31, 1041-1047.

19. Gandhi, T.; Trikha, M.; Santhosh, J.; Anand, S. Development of an expert multitask gadget controlled by voluntary eye movements. Expert Syst. Appl. 2010, 37, 4204-4211.

20. Kherlopian, A.R.; Gerrein, J.P.; Yue, M.; Kim, K.E.; Kim, J.W.; Sukumaran, M.; Sajda, P. Electrooculogram based system for computer control using a multiple feature classification model. In Proceedings of the 28th IEEE EMBS Annual International Conference EMBS'06, New York, NY, USA, August 2006; pp. 1295-1298.

21. Manabe, H.; Fukumoto, M. Full-time wearable headphone-type gaze detector. In Proceedings of the Conference on Human Factors in Computing Systems, Montreal, QC, Canada, April 2006; pp. 1073-1078.

22. Lee, J.; Lee, Y. Saccadic eye-movement system modelling using recurrent neural network. In Proceedings of the International Joint Conference on Neural Networks IJCNN`93, Nagoya, Japan, October 1993; pp. 57-60.

23. Kikuchi, M.; Fukushima, K. Pattern Recognition with Eye Movement: A Neural Network Model. In Proceedings of the International Joint Conference on Neural Networks IJCNN'00, Como, Italy, July 2000; pp. 37-40.

24. Barea, R.; Boquete, L.; Mazo, M.; Lopez, E.; Bergasa, L.M. EOG guidance of a wheelchair using neural networks. In Proceedings of 15th International Conference on Pattern Recognition, Barcelona, Spain, September 2000; Volume 4, pp. 668-671.

25. Güven, A.; Kara, S. Classification of electro-oculogram signals using artificial neural network. Expert Syst. Appl. 2006, 31, 199-205.

26. Shuyan, H.; Gangtie, Z. Driver drowsiness detection with eyelid related parameters by Support Vector Machine. Expert Syst. Appl. 2009, 36, 7651-7658.

27. Patmore, W.D.; Knapp B.R. Towards an EOG-based eye tracker for computer control. In Proceedings of the Third International ACM Conference on Assistive Technologies, Marina del Rey, CA, USA, April 1998; pp. 197-203.

28. Usakli, A.B.; Gurkan, S. Design of a novel efficient human-computer interface: An electrooculagram based virtual keyboard. IEEE Trans. Instrum. Meas. 2009, 59, 2099-2108.

29. Bulling, A.; Ward, J.; Gellersen, H.; Tröster, G. Robust recognition of reading activity in transit using wearable electrooculography. In Proceedings of Pervasive Computing 6th International Conference, Pervasive 2008 Sydney, Australia, May 19-22, 2008; pp. 19-37. 
30. Bulling, A.; Roggen, D.; Tröster, G. Wearable EOG goggles: Eye-based interaction in everyday environments. In Proceedings of the 27th International Conference on Human Factors in Computing Systems: Extended Abstracts, Boston, MA, USA, April 2009; pp. 3259-3264.

31. Estrany, B.; Fuster, P.; Garcia, A.; Luo, Y. Human computer interface by EOG tracking. In Proceedings of the 1st International Conference on PErvasive Technologies Related to Assistive Environments, Athens, Greece, July 2008.

32. Vehkaoja, A.T.; Verho, J.A.; Puurtinen, M.M.; Nojd, N.M.; Lekkala, J.O.; Hyttinen, J.A. Wireless head cap for EOG and facial EMG measurements. In Proceedings of the 27th Annual International Conference of the Engineering in Medicine and Biology Society, Shanghai, China, September 2005; pp. 5865-5868.

33. Vuzix Wrap 230 Video Eyewear. Available online: http://www.vuzix.com/iwear/ products_wrap230.html (accessed on 12 December 2010).

34. Winter, B.B.; Webster, J.G. Driven-right-leg circuit design. IEEE Trans. Biomed. Eng. 1983, 30, $62-66$.

35. Brechet, L.; Lucas M.F.; Doncarli; C.; Farina, D. Compression of biomedical signals with mother wavelet optimization and best-basis wavelet packet selection. IEEE Trans. Biomed. Eng. 2007, 54, 2186-2192.

36. Huang, G.B.; Zhu, Q.Y.; Siew, C.K. Extreme learning machine: Theory and applications. Neurocomputing 2006, 70, 489-501.

37. Huang, G.B.; Siew, C.K. Extreme learning machine with randomly assigned RBF kernels. Int. J. Inf. Technol. 2005, 11, 16-24.

38. Deng, L.Y.; Hsu, C.-L.; Lin, T.-C.; Tuan, J.-S.; Chang, S.-M. EOG-based Human-Computer Interface system development. Expert Syst. Appl. 2010, 37, 3337-3343.

39. Bhandari, A.; Khare, V.; Santhosh, J.; Anand, S. Wavelet based compression technique of Electro-oculogram signals. In Proceedings of the 3rd International Conference on Biomedical Engineering, Kuala Lumpur, Malaysia, December 2006; Volume 15, Part 10, pp. 440-443.

40. Barea, R.; Boquete, L.; Mazo, M.; Lopez E.; Bergasa, L.M. E.O.G. guidance of a wheelchair using spiking neural networks. In Proceedings of the European Symposium on Artificial Neural Networtks, Bruges, Belgium, April 2000; pp. 233-238.

41. Liu, C-C.; Chaovalitwongse, W.A; Pardalos, P.M.; Seref, O.; Xanthopoulos, P.; Sackellares, J.C.; Skidmore, F.M. Quantitative analysis on electrooculography (EOG) for neurodegenerative disease. In Proceedings of the Conference on Data Mining, Systems Analysis, and Optimization in Biomedicine, Gainesville, FL, USA, March 2007; pp. 246-253.

42. Bulling, A.; Roggen, D.; Tröster, G. EyeMote-Towards context-aware gaming using eye movements recorded from wearable electrooculography. In Proceedings of Second International Conference Fun and Games, Eindhoven, The Netherlands, October 20-21, 2008; pp. 33-45.

43. Bulling, A.; Roggen, D.; Tröster, G. It's in your eyes: Towards context-awareness and mobile HCI using wearable EOG goggles. In Proceedings of the 10th International Conference on Ubiquitous Computing, Seoul, South Korea, September 2008; pp. 84-93.

44. Chi, C.; Lin, C. Aiming accuracy of the line of gaze and redesign of the gaze-pointing system. Percept Mot. Skills 1997, 85, 1111-1120. 
45. Itakura, N.; Sakamoto, K. A new method for calculating eye movement displacement from AC coupled electro-oculographic signals in head mounted eye-gaze input interfaces. Biomed. Signal Process Contr. 2010, 5, 142-146.

(C) 2010 by the authors; licensee MDPI, Basel, Switzerland. This article is an open access article distributed under the terms and conditions of the Creative Commons Attribution license (http://creativecommons.org/licenses/by/3.0/). 